\title{
An asymmetric synthesis of all stereoisomers of piclavines A1-4 using an iterative asymmetric dihydroxylation
}

\author{
Yukako Saito, Naoki Okamoto and Hiroki Takahata*
}

\section{Full Research Paper}

\section{Address:}

Faculty of Pharmaceutical Sciences, Tohoku Pharmaceutical

University, Sendai 981-8558, Japan

\section{Email:}

Yukako Saito - yukako-s@tohoku-pharm.ac.jp; Naoki Okamoto okamotonak@chugai-pharm.co.jp; Hiroki Takahata* -

takahata@tohoku-pharm.ac.jp

* Corresponding author

\section{Open Access}

Beilstein Journal of Organic Chemistry 2007, 3, No. 37 doi:10.1186/1860-5397-3-37

Received: 05 September 2007

Accepted: 29 October 2007

Published: 29 October 2007

(C) 2007 Saito et al; licensee Beilstein-Institut

License and terms: see end of document.

\section{Abstract}

The asymmetric synthesis of both enantiomers of piclavines A1, A2, A3, and A4 has been achieved using an iterative asymmetric dihydroxylation with enantiomeric enhancement.

\section{Background}

Indolizidine units are frequently found in many natural products and designed bioactive molecules. [1] Among these alkaloids, piclavines A1-4 (Figure 1), extracted from the tunicate Clavelina picta and the first indolizidine alkaloids to be found in the marine biosphere, exhibit interesting antimicrobial activities. [2] However, very little effort has been made to synthesize the piclavines. So far, among the four isomers shown in Figure 1, the synthesis of piclavine A4 [3] and a mixture of piclavines A1 and A2 [4] has been reported, but the synthesis of all four isomers has never been reported. In addition, their biological activities have been evaluated as a mixture of piclavines A1-4. [2] Therefore, we were inspired to develop a comprehensive synthetic program for these alkaloids.

The asymmetric synthesis of an indolizidine ring remains a great challenge. Our interest in this field has been focused on potential strategies based on the enantiomeric enhancement
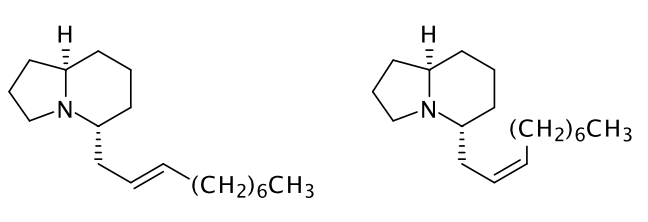

1 piclavine $A 1$

2 piclavine $A 2$
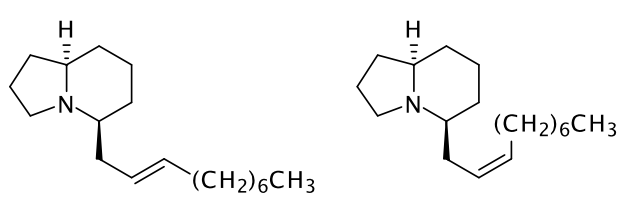

3 piclavine $A 3$

4 piclavine $A 4$ 


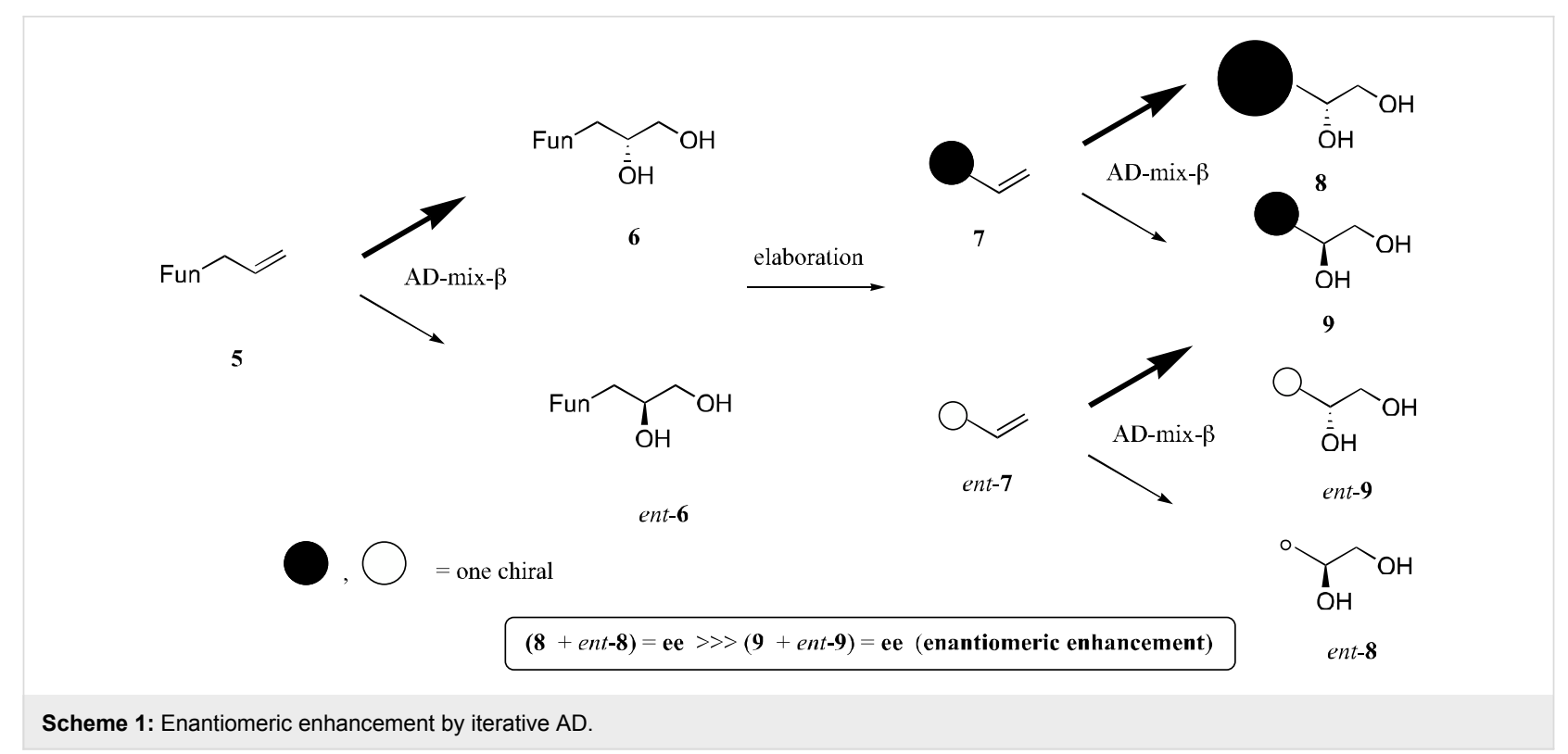

caused by the twofold or more application of the Sharpless asymmetric dihydroxylation (AD) [5,6] or Brown's asymmetric allylboration[7] reactions. In general, the enantiomeric excesses (ees) obtained for $\mathrm{AD}$ of terminal olefins are lower than for trans disubstituted olefins. However, it is expected that iterative $\mathrm{AD}$ terminal olefins will give products with high ees based on the following consideration. The first AD (AD-mix- $\beta$ ) of 5 produces major and minor enantiomers, $\mathbf{6}$ and ent-6, which are elaborated by introduction of terminal olefins to afford 7 and ent-7, respectively. The second $\mathrm{AD}$ of a mixture of 7 and ent-7 provides four products $\mathbf{8}, \mathbf{9}$, ent-9, and ent-8. The relationship between $\mathbf{8}$ and $\mathbf{9}$ is diastereomeric. Since very little of the mirror image compound ent-8 is prepared, the ee of the major product 8 will be very high. On the other hand, the ee of the minor diastereomer 9 or ent $\mathbf{9}$ will be a low (Scheme 1). In most cases, when the products prepared by the iterative AD are acyclic and their asymmetric centers are remote, it is difficult to separate two diastereomers. Since transformation of acyclic compounds to cyclic derivatives provides rigid conformation and causes close proximity between two chiral centers, it is expected to greatly facilitate separation of two diastereomers. In this line, we report a full paper describing a new synthesis of all stereoisomers of piclavines A1-4 with high enantiomeric purity (amplification) for the major diastereomer via iterative AD reaction of terminal olefins. [8]

\section{Results}

Actually we developed a general access to 5-substituted indolizidines 10 (all four stereoisomers of indolizidine 209D) with high enantio-enhancement (92-98\% ee) via a sequence of iterative AD reactions starting from an achiral $\mathrm{N}$-pentenylphthalimide (11). [9] The two stereogenic centers in
10 were constructed with high enantio-enhancement via a sequence of twofold AD reactions as shown in Scheme 2.

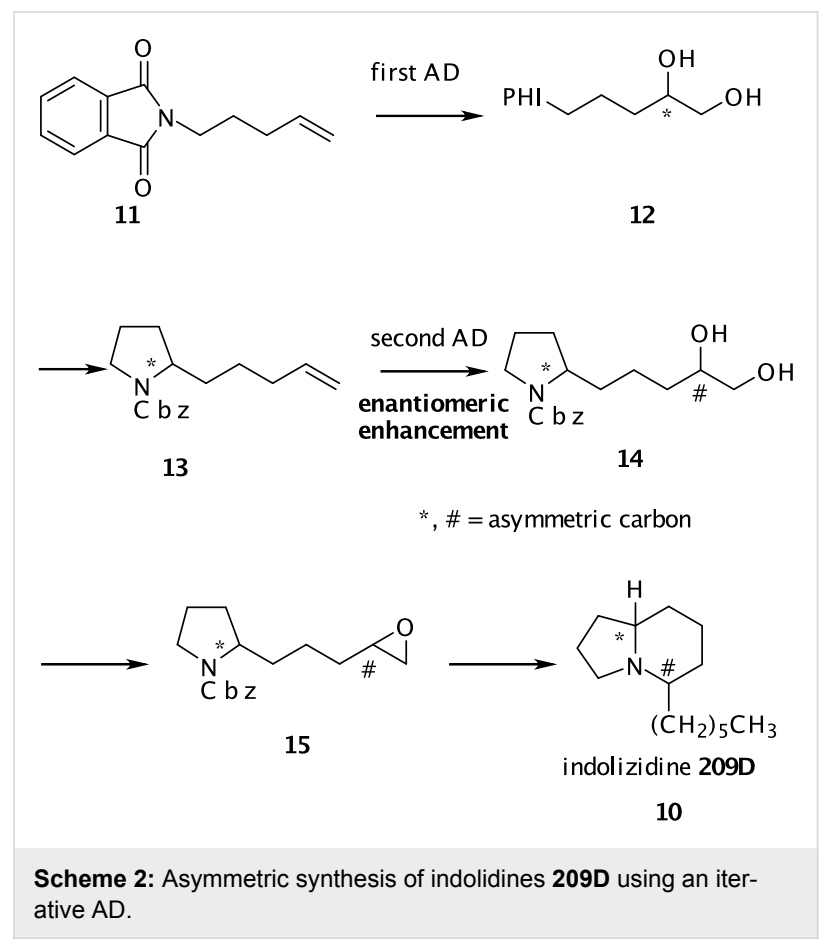

We embarked on the synthesis of piclavines A1-4 using the four stereoisomers of the epoxides 15[9] derived from 11 according to our reported procedure as synthetic intermediates. Regioselective cleavage of the epoxide (2R-[4S])-15 rings with lithium acetylide generated from 1 -nonyne with $n$-butyl lithium in combination with $\mathrm{BF}_{3}-\mathrm{Et}_{2} \mathrm{O}[10]$ gave the secondary alcohols (2R-[4S])-16 in $94 \%$. It is impossible to utilize hydrogenolysis 


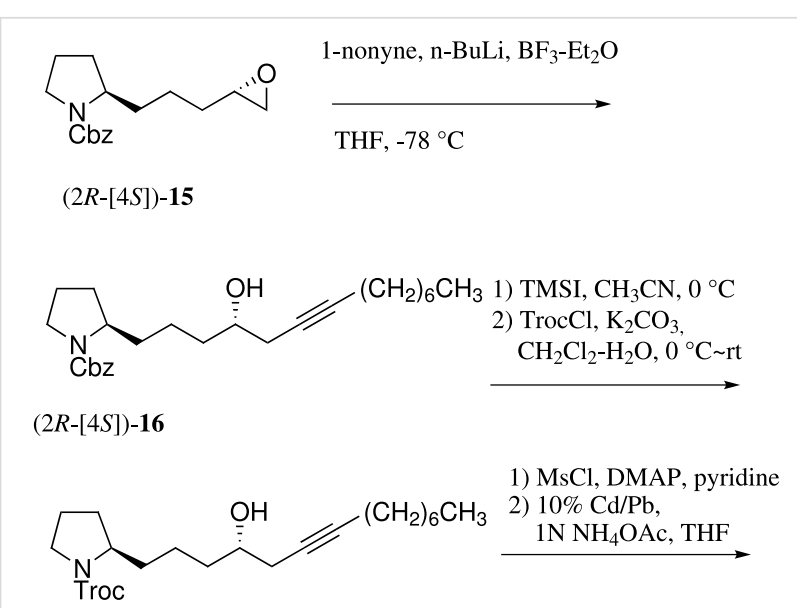

$(2 R-[4 S])-\mathbf{1 7} *$ Only major diastereomers $(\mathbf{1 5}, \mathbf{1 6}$, and $\mathbf{1 7})$ are shown.

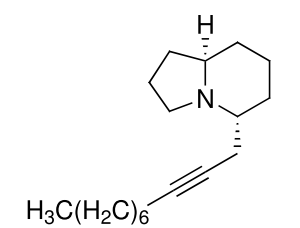

$(5 R, 8 \mathrm{a} R)-18$

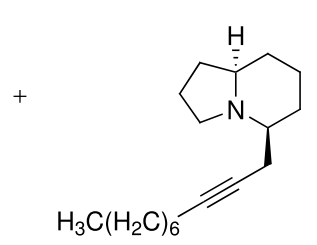

$(5 S, 8 \mathrm{a} R)-\mathbf{1 8}$ \begin{tabular}{l|l}
$\mathrm{Na}$, liq. $\mathrm{NH}_{3}, \mathrm{THF}$ & $5 \% \mathrm{Pd}^{-\mathrm{BaSO}_{4}}(50 \mathrm{~g} \%), \mathrm{THF}$
\end{tabular}

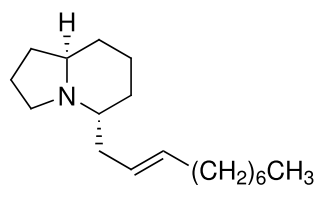

1

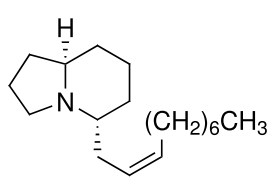

2
Scheme 3: Asymmetric synthesis of piclavines $A 1$ and $A 2$ from $(2 R-$ [4S])-15.

due to reactivity of the acetylene unit. Indeed, the $N$-protecting group exchange of benzyloxycarbonyl (Cbz) for 2,2,2-trichloroethoxycarbonyl (Troc) in $\mathbf{1 7}$ was examined. The use of basic reagents such as $\mathrm{Ba}(\mathrm{OH})_{2}[11]$ and $\mathrm{KOH}[12]$ failed to afford clean deprotection of Cbz. However, treatment of (2R-[4S])-16 with iodotrimethylsilane (TMSI)[13] in $\mathrm{CH}_{3} \mathrm{CN}$ provided the amine $(75 \%)$, which was treated with $\mathrm{TrocCl} / \mathrm{K}_{2} \mathrm{CO}_{3}$ to afford the Troc carbamates $(2 R-[4 S])-\mathbf{1 7}$. After mesylation of $[2 S-$ $(4 R)$ ]-17, $N$-deprotection of the resulting mesylate with $10 \%$ $\mathrm{Cd}-\mathrm{Pb}[14]$ gave the desired $(5 R, 8 \mathrm{a} R)-\mathbf{1 8}\left\{[\alpha]^{25} \mathrm{D}+9.03(c 0.32\right.$, $\left.\left.\mathrm{CH}_{3} \mathrm{OH}\right)\right\}$ as a major product and $(5 R, 8 \mathrm{a} S)-\mathbf{1 8}\left\{[\alpha]^{25} \mathrm{D}-22.7(c\right.$ $\left.\left.1.54, \mathrm{CH}_{2} \mathrm{Cl}_{2}\right)\right\}$ as a minor product in a ratio of $2.3: 1$ in $25 \%$ overall yield. As expected, at this stage it was possible to separate the two diastereomers because transformation of monocyclic compounds (pyrrolidines) to bicyclic derivatives (indolizidines) provides rigid conformation and causes close proximity (a change from 1,5- to 1,3-relationship) between the

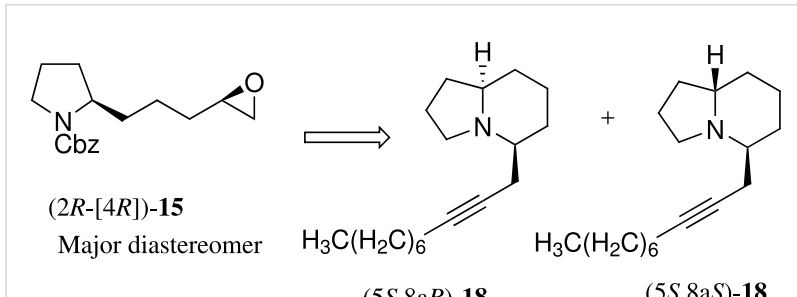

$(5 S, 8 \mathrm{a} R)-18$

$(5 S, 8 \mathrm{a} S)-\mathbf{1 8}$

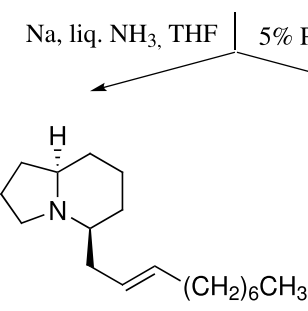

3

Scheme 4: Asymmetric synthesis of piclavines A3 and A4 from (2R[4R])-15.

two asymmetric centers. With indolizidine $(5 R, 8 \mathrm{a} R)-\mathbf{1 8}$ in hand, we examined partial-reduction of their triple bonds. First, treatment of $(5 R, 8 \mathrm{a} R)-\mathbf{1 8}$ with sodium in liquid ammonia gave the desired piclavine A1 (1) $\left\{[\alpha]^{25} \mathrm{D}-5.6\left(c 0.84, \mathrm{CH}_{2} \mathrm{Cl}_{2}\right)\right\}$ containing a trans-olefin in $71 \%$ yield. Exposure of hydrogen to $(5 R, 8 \mathrm{a} R)-18$ in the presence of Lindlar catalyst $\left(\mathrm{Pd} / \mathrm{CaCO}_{3} / \mathrm{Pb}\right)$ or Rosenmund catalyst $\left(5 \% \mathrm{Pd}-\mathrm{BaSO}_{4}\right)$ was carried out in order to obtain a cis olefin product. However, hydrogenation using 10 $\mathrm{g} \%$ catalysts scarcely proceeded, because the tertiary amine in indolizidine presumably works as a poison of the catalyst. Accordingly, the use of a large amount (50 g\%) of $5 \%$ $\mathrm{Pd}-\mathrm{BaSO}_{4}$ took place with semi-reduction of $(5 R, 8 \mathrm{a} R)-\mathbf{1 8}$ to provide piclavine A2 (2) $\left\{[\alpha]^{25} \mathrm{D}+4.03\left(c 0.21 \mathrm{CH}_{2} \mathrm{Cl}_{2}\right)\right\}$ in $53 \%$ yield (Scheme 3 ). The ${ }^{1} \mathrm{H}$ and ${ }^{13} \mathrm{C}$ NMR spectra of synthetic piclavines $\mathrm{A} 1$ and $\mathrm{A} 2$ are in good agreement with those of a mixture of natural piclavines A1 and A2. [2]

A similar sequence of the epoxide $(2 R-[4 R])-15$ prepared by (DHQD) $)_{2}$-PYR ligand-induced AD reaction of $(R)$-13 afforded the desired $(5 S, 8 \mathrm{a} R)-\mathbf{1 8}\left\{[\alpha]^{25} \mathrm{D}-67.5\left(c 1.11, \mathrm{CH}_{2} \mathrm{Cl}_{2}\right)\right\}$ as a major product and $(5 S, 8 \mathrm{a} S)-18\left\{[\alpha]^{25} \mathrm{D}^{-3.11}\left(c 0.62, \mathrm{CH}_{3} \mathrm{OH}\right)\right\}$ as a minor product in a ratio of 3.6:1 in $46 \%$ overall yield from (2R-[4R])-15. Similar semi-reduction of $(5 S, 8 \mathrm{a} R)-\mathbf{1 8}$ with $\mathrm{Na} /$ $\mathrm{NH}_{3}$ and $\mathrm{H}_{2} / 10 \% \mathrm{Pd}-\mathrm{BaSO}_{4}$ gave piclavine A3 (3) (76\%) $\{[\alpha]$ ${ }^{25}{ }_{\mathrm{D}}-74.3\left(c\right.$ 1.30, $\left.\mathrm{CH}_{2} \mathrm{Cl}_{2}\right)$ and piclavine $\mathrm{A} 4(4)[\alpha]^{25} \mathrm{D}-76.5$ (c $\left.\left.0.63 \mathrm{CH}_{2} \mathrm{Cl}_{2}\right)\right\}$ lit. [3] $\left.[\alpha]^{20}{ }_{\mathrm{D}}-74.8\left(c 0.5 \mathrm{CH}_{2} \mathrm{Cl}_{2}\right)\right\}$ in $84 \%$ yield, respectively (Scheme 4). Spectral data of 4 were completely consistent with values reported. [3]

With two diastereomers (2S-[4R])- and (2S-[4S])-15 in hand, [9] the enantiomers of piclavines A1-4 were prepared according to the procedure described above (Scheme 5). However, the abso- 
lute configuration of natural products remains unassigned. [2]

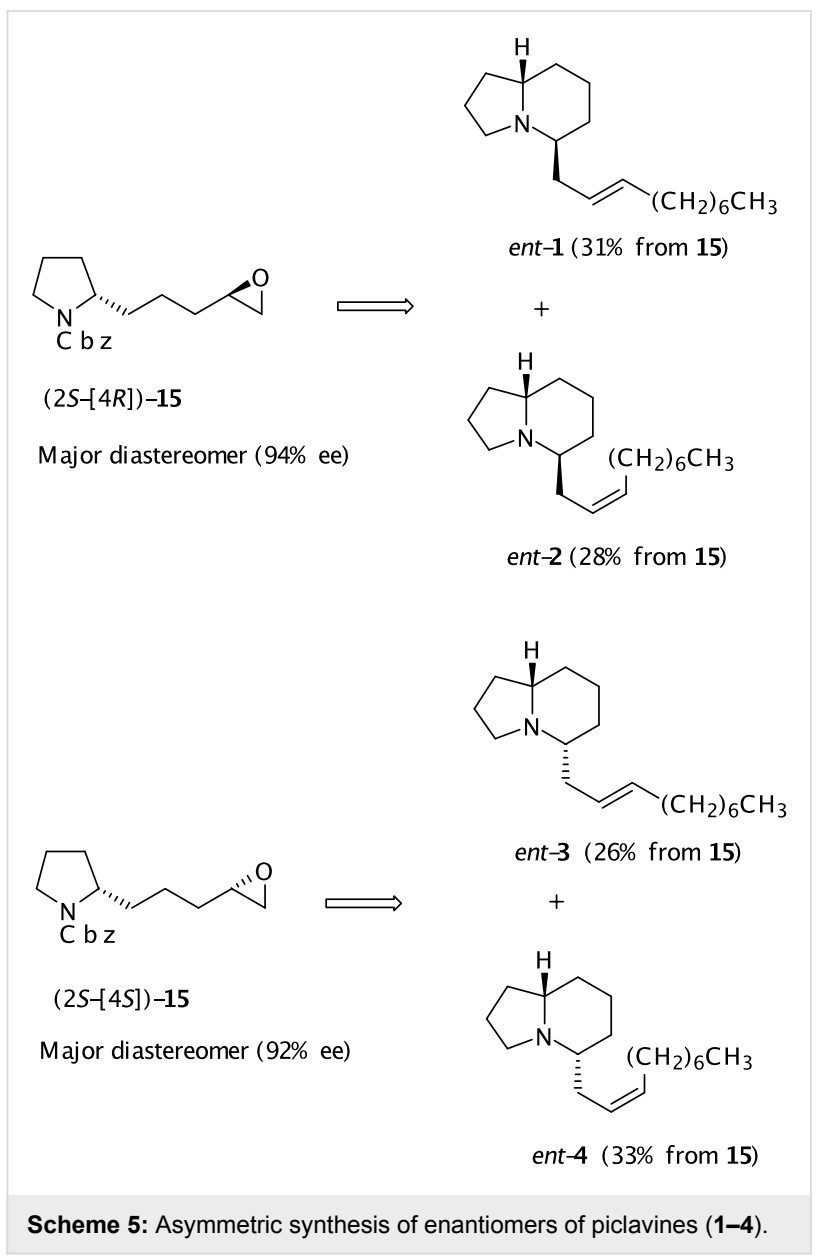

\section{Conclusion}

In summary, we accomplished the asymmetric synthesis of both enantiomers of piclavines A1, A2, A3, and A4 with high enantio-enhancement via iterative $\mathrm{AD}$ reactions starting from an achiral $N$-pentenylphthalimide $\mathbf{1 1}$.

\section{Supporting Information}

\section{Supporting Information File 1}

Synthetic details, spectral properties and HRMS data. Experimental details for an asymmetric synthesis of all stereoisomers of piclavines A1-4 using an iterative asymmetric dihydroxylation. Experimental data which includes experimental details on the spectral instruments. [http://www.beilstein-journals.org/bjoc/content/ supplementary/1860-5397-3-37-S1.doc]

\section{Acknowledgments}

This work was supported in part by the High Technology Research Program from the Ministry of Education, Sciences, Sports and Culture of Japan

\section{References}

1. Michael, J. P. Nat. Prod. Rep. 2007, 24, 191-222. doi:10.1039/ b509525p

2. Raub, M. F.; Cardellinia, J. H., II; Spande, T. F. Tetrahedron Lett. 1992, 33, 2257-2260. doi:10.1016/S0040-4039(00)74183-9

3. Jefford, C. W.; Sienkiewicz, K.; Thornton, S. R. Helv. Chim. Acta 1995, 78, 1511-1524. doi:10.1002/hlca.19950780610

4. McAlonan, H.; Potts, D.; Stevenson, P. J.; Thompson, N. Tetrahedron Lett. 2000, 41, 5411-5414. doi:10.1016/S0040-4039(00)00868-6

5. Takahata, H. J. Synth. Org. Chem., Jpn. 1999, 57, 835-844.

6. Takahata, H. Trends Org. Chem. 2000, 8, 101-119.

7. Takahata, H.; Saito, Y.; Ichinose, M. Org. Biomol. Chem. 2006, 4, 1587-1595. doi:10.1039/b601489e

8. Takahata, H.; Okamoto, N. Bioorg. Med. Chem. Lett. 2000, 10, 1799-1801. doi:10.1016/S0960-894X(00)00341-3

9. Takahata, H.; Kubota, M.; Ihara, K.; Okamoto, N.; Momose, T.; Azer, N.; Eldefrawi, A. T.; Eldefrawi, M. E. Tetrahedron: Asymmetry 1998, 9 , 3289-3301. doi:10.1016/S0957-4166(98)00338-3

10. Yamaguchi, M.; Nobayashi, Y.; Hirao, I. Tetrahedron Lett. 1983, 24, 5121-5122. doi:10.1016/S0040-4039(00)94057-7

11. Overman, L. E.; Sharp, M. J. Tetrahedron Lett. 1988, 29, 901-904. doi:10.1016/S0040-4039(00)82477-6

12. Angle, S. R.; Arnaiz, D. O. Tetrahedron Lett. 1989, 30, 515-518. doi:10.1016/S0040-4039(00)95241-9

13. Ihara, M.; Taniguchi, N.; Noguchi, K.; Fukumoto, K.; Kametani, T. J. Chem. Soc., Perkin Trans. 1 1988, 1277-1281. doi:10.1039/ p19880001277

14. Dong, Q.; Anderson, C. E.; Ciufolini, M. A. Tetrahedron Lett. 1995, 36, 5681-5682. doi:10.1016/0040-4039(95)01122-X

\section{License and Terms}

This is an Open Access article under the terms of the Creative Commons Attribution License (http://creativecommons.org/licenses/by/2.0), which permits unrestricted use, distribution, and reproduction in any medium, provided the original work is properly cited.

The license is subject to the Beilstein Journal of Organic Chemistry terms and conditions:

(http://www.beilstein-journals.org/bjoc)

The definitive version of this article is the electronic one which can be found at: $\underline{\text { doi:10.1186/1860-5397-3-37 }}$ 